\title{
The Effect of Topical Tranexamic Acid on Intraoperative Blood Loss in Patients Undergoing Posterior Lumbar Laminectomy and Discectomy: A Randomized, Double-Blind, Controlled Trial Study
}

\author{
Gholamreza Farzanegan ${ }^{1}$, Fathollah Ahmadpour ${ }^{1}$, Hadi Khoshmohabbat ${ }^{1}$, \\ Masoud Khadivi ${ }^{2}$, Hamid Reza Rasouli ${ }^{1}$, Mohammad Eslamian ${ }^{3}$ \\ ${ }^{1}$ Trauma Research Center, Baqiyatallah University of Medical Sciences, Tehran, Iran \\ ${ }^{2}$ Spine Center of Excellence, Yas Hospital, Tehran University of Medical Sciences, Tehran, Iran \\ ${ }^{3}$ Department of Neurosurgery, Shariati Hospital, Tehran University of Medical Sciences, Tehran, Iran
}

\section{Study Design: Randomized, double-blind, controlled trial study.}

Purpose: This study aimed to evaluate the safety and efficacy of topical tranexamic acid (TXA) on intraoperative blood loss (IBL) in patients that have degenerative lumbar canal stenosis and undergo posterior lumbar laminectomy and discectomy.

Overview of Literature: The volume of IBL is directly proportional to potential surgical complications. Recent reports have shown that the topical use of antifibrinolytic drugs, such as TXA, during surgery might decrease IBL and improve patient outcomes.

Methods: A total of 104 patients with lumbar canal stenosis were enrolled in this randomized, double blinded clinical trial. Participants were randomized and divided into two groups: TXA (54 cases) and control (50 cases). In the TXA group, a TXA solution was used for washing and soaking, whereas, in the control group, irrigation of wound was with normal saline. IBL, pre- and postoperative coagulative studies, operation time, conventional hemostatic agent usage, systemic complications, and length of hospitalization were consecutively recorded. All participants were followed for an additional two months to gather data on their recovery status and time to return to work (RTW).

Results: At baseline, there was no difference in clinical or lab findings, between the groups. IBL and use of hemostatic agents were significantly decreased in TXA group, as compared to the control group ( $p=0.001$ and $p=0.011$, respectively). Systemic complications, length of hospitalization, and RTW were not significantly different between groups $(p=0.47, p=0.38$, and $p=0.08$, respectively).

Conclusions: This study showed that topical use of TXA during surgery may decrease IBL and minimize the use of hemostatic materials during posterior midline-approach laminectomy and discectomy, without increasing the potential for complications seen with intravenous TXA usage.

Keywords: Tranexamic acid; Blood loss; Topical; Laminectomy; Complications

Received Jul 18, 2021; Revised Nov 6, 2021; Accepted Nov 9, 2021

Corresponding author: Mohammad Eslamian

Department of Neurosurgery, Shariati Hospital, Tehran University of Medical Sciences, Tehran, Iran

Tel: +98-9188153139, Fax: +98-21-88003539, E-mail: meslamian@student.tums.ac.ir 


\section{Introduction}

High volume of intraoperative blood loss (IBL) during any spine operation is a major dilemma for surgeons $[1,2]$. Large skin incisions and a need to access multiple levels, in addition to the extensive involvement of soft tissue and bone removal during posterior-approach spinal surgeries are all possible factors that increase IBL [2-4]. Extensive IBL can lead to the complications such as hypotension, metabolic acidosis, and coagulopathies and can negatively impact surgical outcomes [5,6]. In recent decades, many efforts have been made to decrease IBL and improve clinical outcomes in patients undergoing spinal surgeries [3-8]. Reported potential strategies include the use of smaller incisions, less manipulation of soft tissue and bone, the use of various hemostatic agents during surgery, the induction of controlled hypotension, and systemic or topical use of antifibrinolytic agents [4].

The safety and efficacy of topical tranexamic acid (TXA) in orthopedic surgeries (such as knee and pelvic surgeries), hepatic surgeries, and cardiac surgeries has been shown previously [9-12]. TXA is a synthetic lysine analog that acts by reversible binding to tissue plasminogen activator, plasminogen, and plasmin, thus preventing their attachment to fibrin. This leads to prevention of clot lysis. TXA can cross the blood-brain barrier and concentration studies have shown that the cerebrospinal fluid levels of TXA is one-tenth of its blood level, 2 hours after intravenous injection of $10 \mathrm{mg} / \mathrm{kg}$ of TXA [13]. Systemic use of TXA, however, has possible complications such as deep venous thrombosis, pulmonary embolism, and seizure. Colomina et al. [14] suggests the topical use of TXA, as an alternative to intravenous use, and shows that the administration of topical TXA has the potential to reduce blood loss in a safe manner in some surgeries (i.e., joint arthroplasty) [14-16].

There are scarce data regarding the topical use of TXA in spinal surgeries. Previous studies have focused on postoperative bleeding [8], and data regarding the role of topical TXA in reduction of IBL is limited to small-size retrospective studies $[15,17]$. The current prospective, randomized double-blind clinical trial, aimed to evaluate the safety and effectiveness of topical TXA in the reduction of IBL in patients that underwent standard, open, posterior midline-approach, bilateral laminectomy, and discectomy.

\section{Materials and Methods}

\section{Study design}

A total of 104 patients participated in this randomized, double-blind clinical trial. All participants were referred to Baqiyatallah University Hospital clinic and were candidates for posterior lumbar spinal surgery with the diagnosis of disk herniation, spinal canal stenosis, or both. The diagnosis was made based on history, physical examination, and magnetic resonance imaging. Indication for surgery was determined by a senior, attending, spine surgeon; indications included neurological deficit, neurologic claudication, or intolerable pain that was unresponsive to conservative management. Plain dynamic bending X-rays were performed to identify vertebral instability and the need for possible instrumentation. The goals and course of the study were explained, and all participants signed a written informed consent before surgery. The study was confirmed by institutional review board and ethical committee of Baqiyatallah University of Medical Sciences, registered under the number (IR.BMSU.BAQ.REC.1398.001). It is also registered at the Iranian Registry of Clinical Trials (IRCT): IRCT20200203046351N1.

\section{Inclusion and exclusion criteria}

The participants of the study were chosen according to our inclusion and exclusion criteria and all participants were required to have a history of failed previous therapeutic conservative intervention. The inclusion criteria were as follows: patients aged 18 to 75 years, a diagnosis of lumbar canal stenosis, herniated disk or both, and their condition must have been unresponsive to at least 8 weeks of conservative treatment (i.e., nonsteroidal anti-inflammatory drugs, physiotherapy, and muscle strengthening exercises). In the case of progressive neurologic deficit, or any other emergent condition, the patient was candidate for surgery immediately. History and physical examination were obtained by an attending spine surgeon or fellow. Magnetic resonance imaging was reviewed by an expert radiologist and final indication for the surgery was determined by the senior attending spine surgeon.

Patients with the history of any coagulopathy or taking medication that might influence the coagulation cascade (e.g., aspirin, warfarin, etc.) were excluded from the study. Due to possible disturbances in coagulation lab tests, 
participants that had experienced recent trauma were excluded from the study. Use of TXA in patients with liver/ kidney dysfunction and those with the history of seizure is relatively contraindicated; therefore, they were excluded from the study.

\section{Randomization}

The randomization of the participants completed via Randlist (Datinf GmbH, Stuttgart, Germany) software. In short, 104 random numbers were generated, concealed sequentially in opaque, sealed envelopes, and kept in the operation room. Just before the operation began each participant was assigned a random number, with equal probability. Patients who received an even number were assigned to the intervention (TXA) group and those with odd numbers were assigned to the control group. A surgical technician nurse, who was unaware of the randomization and did not take part in the intervention, prepared the solutions (TXA or saline) used for irrigation during the operation, based on the even or odd code. The surgeon, anesthesia team, and all patients were blind to randomization and were not aware of the ingredients in the solution.

\section{Intervention}

Patients were treated by open, bilateral, posterior laminectomy, and discectomy. The same surgeon performed all of the operations at our university hospital. In order to rule out any confounding influence, the anesthesia team was also the same for all patients. The surgeon incised the skin at the midline and used the bilateral approach for muscle and soft tissue dissection and a bilateral laminectomy at the involved levels was performed. The lower segment of the cranial lamina and the upper part of the caudal lamina were removed, and ligamentum flavum between these parts was dissected. Herniated disc fragments were completely removed, and decompression of the thecal sac and nerve root was achieved. Non-instrumented posterolateral fusion was performed for all the patients. Finally, the wound was closed in separate layers. After random allocation of the subjects (described above), the same surgical technician nurse made an irrigation solution which contained $3 \mathrm{~g}$ of TXA in $100 \mathrm{~mL}$ of normal saline for the TXA group, and only normal saline for the control group. The solution was used during all parts of the surgery. For example, after skin incision, the solution was used during bipolar electrocoagulation. Blunt dissection of the paraspinal muscle was performed by wet sterile gauze with the solution, and after stripping muscles from the bone the surgical field was filled with the wet sterile gauze (soaked in the solution and left for 5 minutes). After laminectomy and final discectomy, the surgical field was filled with the solution and remained for 5 minutes. At the end of the surgery the wound was irrigated with the solution and drain fixed for all the patients.

\section{Outcome measurements}

The primary outcome measure of the study was IBL, which was recorded in milliliters. The duration of surgery and length of postoperative hospital stay were recorded in minutes and days respectively. Postoperative laboratory coagulation tests were performed and consisted of prothrombin time (PT), partial thromboplastin time (PTT) and the international normalized ratio (INR); the results of these tests were compared between the groups. Any systemic complication was recorded. All the subjects were followed for a minimum of 2 months and any delayed complications, as well as time to return to work (RTW), were recorded.

\section{Statistical analysis}

Statistical analyses were performed using SPSS software package ver. 17.0 for Windows (SPSS Inc., Chicago, IL, USA). Data are presented as mean \pm standard deviation. One-sample Kolmogorov-Smirnov was used to check the normal distribution of the measurements. Independent samples $T$-test was carried out to investigate the differences of the quantitative variable between two groups. Pearson's correlation coefficients were calculated to investigate the correlation of age and other quantitative variables with blood loss volume. A chi-square test was used to evaluate the association of diagnosis with the amount of blood loss. Statistical significance was defined as a $p$-value $<0.05$.

\section{Results}

Of 134 patients who were assessed for eligibility between June 2020 and February 2021, 104 participants were randomly assigned to two groups: the TXA group $(n=54)$ and the control group $(n=50)$. None of the patients were lost 


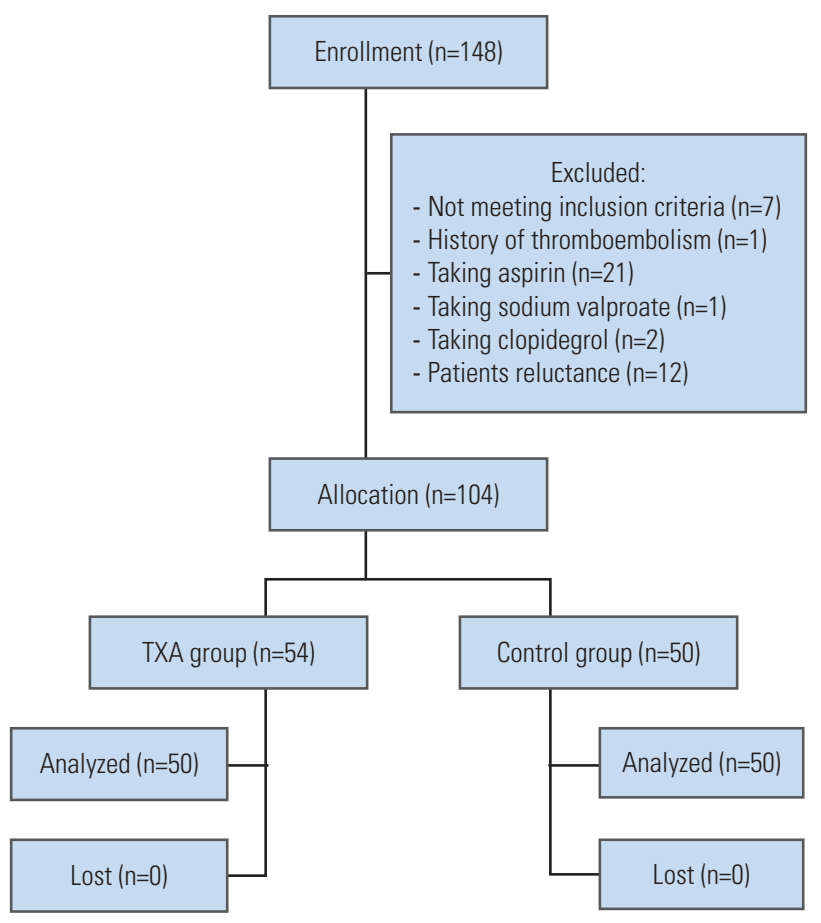

Fig. 1. The flowchart of study progress. TXA, topical tranexamic acid.

during follow-up, and the data for all 104 patients were included in the final analysis (Fig. 1). The characteristics of the participants are provided in Table 1 .

\section{Preoperative measurements}

As it is shown in Table 1, at baseline, there were no significant differences between the groups regarding demographic characteristics and blood lab findings (including renal, hepatic, and coagulation parameters).

\section{Intraoperative measurements}

IBL was significantly lower in TXA group as compared to the control group $(230.00 \pm 165.78$ versus $407.20 \pm 323.58$, respectively; $p<0.001$ ). We also found that the operation time was shorter among patients in TXA group, as compared to the control group, although the difference was not statistically significant $(79.8 \pm 27.6$ versus $83.40 \pm 36$ minutes, respectively; $p=0.62$ ). We found that surgeons had used significantly lower hemostatic material during operation of patients in the TXA group $(p<0.05)$ (Table $2)$. This included all conventional hemostatic materials, such as oxidized cellulose polymers, absorbable gelatin sponges, and bone waxes.

\section{Postoperative measurements}

The statistical analysis showed that there was no significant difference in lab data or coagulation parameters between the groups (Table 2); and the length of hospital stay was similar between the groups $(p=0.38)$. There was not any significant difference in the prevalence of complications between groups, including deep vein thrombosis, pulmonary thromboembolism, urinary tract infection, severe low back pain, dyspnea, intestinal pseudo-obstruction, or coccygeal pain $(p=0.47)$. There was not any occurrence of deep vein thrombosis or pulmonary thromboembolism in either group. At the end of follow-up, the RTW time was evaluated and there were no significant differences between groups (Table 2).

\section{Discussion}

The present double-blind randomized controlled trial was conducted to assess the efficacy and safety of using topical TXA to reduce IBL in standard posterior lumbar laminectomy and fusion surgery. The study showed that using topical TXA significantly reduced IBL; this could provide better visualization of the operation field by the surgeon and a decrease in the use of hemostatic agents, thereby, shortening the operation time and the length of hospital stay. There was no significant difference in age, body mass index, past medical history, past surgical history and drug history, liver function studies, renal function tests, and coagulation tests between two groups; all of which are the most well-known factors to influence intraoperative bleeding. Our results showed a significant decrease in the use of conventional hemostatic agents in TXA group, as compared to the control group. This is in the line with other advantages of reduced intraoperative bleeding.

Since the development of TXA (synthetic lysine), numerous studies have been conducted on the effectiveness of antifibrinolytic compounds $[15,18]$. TXA binds to the tissue plasminogen activator, plasminogen, and plasmin and prevents their attachment to fibrin, thereby inhibiting the lysis of clots. The ability of TXA to cross the bloodbrain barrier may explain the mechanism for the development of serious complications in patients who receive the systemic intravenous form of TXA $[13,19]$.

The exact role of topical TXA on IBL in different surgical procedures needs to be more clearly understood before 
Table 1. Comparison of baseline studied variables

\begin{tabular}{|c|c|c|c|}
\hline Characteristic & Topical TXA group & Control group & $p$-value \\
\hline No. of patients & 54 & 50 & \\
\hline Age (yr) & $51.39 \pm 12.77$ & $54.8 \pm 14.04$ & 0.31 \\
\hline Gender & & & 0.56 \\
\hline Male & 26 & 27 & \\
\hline Female & 28 & 23 & \\
\hline Body mass index $\left(\mathrm{kg} / \mathrm{cm}^{2}\right)$ & $26.57 \pm 4.18$ & $26.56 \pm 5.44$ & 0.98 \\
\hline Diagnosis & & & 0.74 \\
\hline Lumbar disk herniation & 20 & 22 & \\
\hline Lumbar canal stenosis & 14 & 12 & \\
\hline Lumbar disk herniation and canal stenosis & 20 & 16 & \\
\hline Past medical history & & & 0.32 \\
\hline Yes & 8 & 12 & \\
\hline No & 45 & 38 & \\
\hline Past surgical history & & & 0.23 \\
\hline Yes & 19 & 24 & \\
\hline No & 35 & 26 & \\
\hline Drug history & & & 0.82 \\
\hline Yes & 14 & 12 & \\
\hline No & 40 & 38 & \\
\hline Aspartate aminotransferase (U/L) & $27.81 \pm 7.12$ & $26.34 \pm 5.58$ & 0.24 \\
\hline Alanine aminotransferase (IU/L) & $30.78 \pm 6.48$ & $31.06 \pm 5.37$ & 0.81 \\
\hline Alkaline phosphatase (U/L) & $40.61 \pm 8.23$ & $38.62 \pm 8.04$ & 0.22 \\
\hline Blood urea nitrogen (mg/dL) & $25.46 \pm 7.31$ & $24.66 \pm 4.97$ & 0.52 \\
\hline Creatinine (mg/dL) & $1.09 \pm 0.17$ & $1.06 \pm 0.12$ & 0.23 \\
\hline Preoperative prothrombin time (sec) & $13.31 \pm 0.77$ & $13.20 \pm 0.53$ & 0.27 \\
\hline Preoperative partial thromboplastin time (sec) & $29.65 \pm 3.24$ & $29.78 \pm 3.22$ & 0.38 \\
\hline Preoperative international normalized ratio & $1.03 \pm 0.07$ & $1.02 \pm 0.06$ & 0.43 \\
\hline
\end{tabular}

Values are presented as number or mean \pm standard deviation.

TXA, topical tranexamic acid.

considering such measures in standard practice. To date, studies have evaluated the efficacy of TXA in orthopedic surgeries, including knee and pelvic surgeries; it has also been evaluated in cardiac surgeries [9]. In case of spinal surgery, the efficacy of intravenous TXA among patients who underwent posterior lumbar surgeries has been studied $[5,15,17,20]$. Elwatidy et al. [21] found that prophylactic intravenous administration of TXA could reduce intraoperative bleeding. Some studies compared the efficacy of high-dose and low-dose intravenous TXA [22]. In addition, the effectiveness of systemic TXA, in single level versus multilevel posterior spine operations [23,24], has been compared. Beynon et al. [22] in 2019 showed that intravenous TXA could reduce IBL when the patient was treated with oral anticoagulants and required emergency spinal surgery. In two systematic reviews, Hui et al. [7] in 2017 and Hariharan et al. [17] in 2019 showed that an intravenous TXA injection was useful in reduction of IBL and operation time; they also mentioned that only highdose TXA could decrease the need for blood transfusions. In a review of studies on intravenous TXA, Yoo et al. [25] found that although the benefits of intravenous injection are significant, the potential side effects of the systemic use of TXA limits the dose of administration. In order to prevent the side effects of intravenous TXA, in recent years, efforts have been made to show the effectiveness of 
Table 2. Comparison of intra- and postoperative variable

\begin{tabular}{|c|c|c|c|}
\hline Variable & Topical TXA group & Control group & $p$-value \\
\hline No. of patients & 54 & 50 & \\
\hline Intraoperative blood loss (mL) & $230.00 \pm 323.58$ & $407.20 \pm 165.77$ & 0.001 \\
\hline Operation time (min) & $79.80 \pm 27.60$ & $83.40 \pm 36$ & 0.62 \\
\hline Involved levels & $1.81 \pm 0.91$ & $2.16 \pm 0.97$ & 0.065 \\
\hline Hemostatic materials & & & 0.011 \\
\hline No & 39 & 23 & \\
\hline Oxidized cellulose & 14 & 23 & \\
\hline Bone wax & 0 & 4 & \\
\hline Gelatin sponge & 1 & 0 & \\
\hline Postoperative prothrombin time (sec) & $13.44 \pm 1.05$ & $13.36 \pm 0.85$ & 0.66 \\
\hline Postoperative partial thromboplastin time (sec) & $30.37 \pm 3.71$ & $30.04 \pm 3.84$ & 0.66 \\
\hline Postoperative international normalized ratio & $1.03 \pm 0.09$ & $1.03 \pm 0.07$ & 0.63 \\
\hline Systemic complications & & & 0.47 \\
\hline No & 45 & 37 & \\
\hline Yes & 9 & 13 & \\
\hline Hospital stay (day) & $1.24 \pm 0.55$ & $1.34 \pm 0.59$ & 0.38 \\
\hline Return to work (day) & $34.50 \pm 10.80$ & $36.90 \pm 20.70$ & 0.08 \\
\hline
\end{tabular}

Values are presented as number or mean \pm standard deviation.

TXA, topical tranexamic acid.

topical TXA. Topical TXA was first used in knee joints surgery with acceptable results $[15,16]$.

To the best of our knowledge, limited studies have focused on the topical use of TXA, and most are limited to postoperative bleeding. Yerneni et al. [8] reported the beneficial effects of topical TXA including a reduction in postoperative bleeding and length of hospital stay. A review by Winter et al. [26] pointed to only two clinical trials on topical TXA, wherein TXA solution was poured into the wound just before closure and of postoperative bleeding was examined $[27,28]$.

Our results showed that IBL was lower in the topical TXA group, as compared to the placebo group (Fig. 1). This finding emphasizes the key role of topical TXA in the reduction of blood loss during surgery; this can result in better visualization, less operation time, and a decrease in surgical complications. Our results are in agreement with the recent study, which demonstrated that topical TXA can decrease IBL in the surgical treatment of thoracic burst fracture [29].

According to the current study, there was no significant difference in complication rate or type between the two groups. We also observed none of the known systemic
TXA-related complications in patients who received topical TXA. Such findings are in line with other studies showing that topical TXA can be administered in maximum doses, while avoiding serious complications of systemic TXA, in thoracolumbar fractures [30]. There was no association between the use of topical TXA and the duration of hospital stay or RTW. The absence of these associations between the groups indicates that other factors, such as type of surgery and preoperative comorbidities, might play the major role in determining hospital stay and recovery. Both groups of the present study underwent posterolateral lumbar fusion surgery and had similar perioperative comorbidities. Postoperative PT, PTT, and INR were not statistically different between groups, which might be explained by relatively moderate bleeding in both groups.

The need for conventional hemostatic agents (e.g., oxidized cellulose polymers, absorbable gelatin sponges, and bone waxes) was lower in the topical TXA group, as compared to the control group. This could be indicative of the topical TXA acting to reduce bleeding not only from soft tissue, but also bleeding from the bones [8]. These findings could imply that topical TXA may be beneficial 
in decreasing the burden of the operations, although this warrants further study.

The main limitation of this study was in determining the optimum topical TXA dose; we calculated dosing based on previous studies that mainly investigated postoperative bleeding. Studies are needed to compare different topical TXA doses, thus determining a safe and effective dose for the purpose of decreasing IBL in various spinal operations. Another limitation of the present study was the difference in the involved levels between the groups, although the difference was not significant. Studies with more stratified groups may be necessary in the future. An additional limitation could be that topical TXA can influence postoperative drainage and total blood loss.; however, previous studies have mainly evaluated the postoperative blood loss and data on this topic has been widely published $[8,26-28]$. In the present study, we focused on the amount of IBL. As the study was aimed to evaluate the relationship between use of TXA and IBL, we did not record functional outcome parameters such as Visual Analog Scale, Oswestry Disability Index, and EuroQol-Five Dimensions. An investigation of patients' functional outcomes, with a longer period for follow-up, is also important for future research.

\section{Conclusions}

Our results illustrate that the decreased amount of IBL in the standard posterior lumbar laminectomy might be associated with administration of topical TXA; we demonstrated this in more than one hundred homogenous participants treated with TXA, as compared to the placebo. This study suggests that topical TXA might be a safe and effective intraoperative medication, but our results may not extend to other diagnoses and surgical procedures, thus, it may merit further studies in this context.

\section{Conflict of Interest}

No potential conflict of interest relevant to this article was reported.

\section{References}

1. Tse EY, Cheung WY, Ng KF, Luk KD. Reducing perioperative blood loss and allogeneic blood transfusion in patients undergoing major spine surgery. J Bone
Joint Surg Am 2011;93:1268-77.

2. Hu SS. Blood loss in adult spinal surgery. Eur Spine J 2004;13(Suppl 1):S3-5.

3. Hofmann A, Ozawa S, Farrugia A, Farmer SL, Shander A. Economic considerations on transfusion medicine and patient blood management. Best Pract Res Clin Anaesthesiol 2013;27:59-68.

4. Lawson JH, Murphy MP. Challenges for providing effective hemostasis in surgery and trauma. Semin Hematol 2004;41(1 Suppl 1):55-64.

5. Willner D, Spennati V, Stohl S, Tosti G, Aloisio S, Bilotta F. Spine surgery and blood loss: systematic review of clinical evidence. Anesth Analg 2016;123:1307-15.

6. Zollo RA, Eaton MP, Karcz M, Pasternak R, Glance LG. Blood transfusion in the perioperative period. Best Pract Res Clin Anaesthesiol 2012;26:475-84.

7. Hui S, Xu D, Ren Z, et al. Can tranexamic acid conserve blood and save operative time in spinal surgeries?: a meta-analysis. Spine J 2018;18:1325-37.

8. Yerneni K, Burke JF, Tuchman A, et al. Topical tranexamic acid in spinal surgery: a systematic review and meta-analysis. J Clin Neurosci 2019;61:1149.

9. Zhang F, Wang K, Li FN, et al. Effectiveness of tranexamic acid in reducing blood loss in spinal surgery: a meta-analysis. BMC Musculoskelet Disord 2014;15:448.

10. Maddali MM, Rajakumar MC. Tranexamic acid and primary coronary artery bypass surgery: a prospective study. Asian Cardiovasc Thorac Ann 2007;15:313-9.

11. Johansson T, Pettersson LG, Lisander B. Tranexamic acid in total hip arthroplasty saves blood and money: a randomized, double-blind study in 100 patients. Acta Orthop 2005;76:314-9.

12. Dalmau A, Sabate A, Acosta F, et al. Tranexamic acid reduces red cell transfusion better than epsilon-aminocaproic acid or placebo in liver transplantation. Anesth Analg 2000;91:29-34.

13. McCormack PL. Tranexamic acid: a review of its use in the treatment of hyperfibrinolysis. Drugs 2012;72:585-617.

14. Colomina MJ, Koo M, Basora M, Pizones J, Mora L, Bago J. Intraoperative tranexamic acid use in major spine surgery in adults: a multicentre, randomized, placebo-controlled trial. Br J Anaesth 2017;118:380- 
90.

15. Li G, Sun TW, Luo G, Zhang C. Efficacy of antifibrinolytic agents on surgical bleeding and transfusion requirements in spine surgery: a meta-analysis. Eur Spine J 2017;26:140-54.

16. Wang J, Wang Q, Zhang X, Wang Q. Intra-articular application is more effective than intravenous application of tranexamic acid in total knee arthroplasty: a prospective randomized controlled trial. J Arthroplasty 2017;32:3385-9.

17. Hariharan D, Mammi M, Daniels K, et al. The safety and efficacy of tranexamic acid in adult spinal deformity surgery: a systematic review and meta-analysis. Drugs 2019;79:1679-88.

18. Tengborn L, Blomback M, Berntorp E. Tranexamic acid: an old drug still going strong and making a revival. Thromb Res 2015;135:231-42.

19. Astedt B. Clinical pharmacology of tranexamic acid. Scand J Gastroenterol Suppl 1987;137:22-5.

20. Wong J, El Beheiry H, Rampersaud YR, et al. Tranexamic acid reduces perioperative blood loss in adult patients having spinal fusion surgery. Anesth Analg 2008;107:1479-86.

21. Elwatidy S, Jamjoom Z, Elgamal E, Zakaria A, Turkistani A, El-Dawlatly A. Efficacy and safety of prophylactic large dose of tranexamic acid in spine surgery: a prospective, randomized, double-blind, placebo-controlled study. Spine (Phila Pa 1976) 2008;33:2577-80.

22. Beynon C, Olivares A, Gumbinger C, Younsi A, Zweckberger K, Unterberg AW. Management of spinal emergencies in patients on direct oral anticoagulants. World Neurosurg 2019;131:e570-8.

23. Xue P, Yang J, Xu X, Liu T, Huang Y, Qiao F, Huang X.
The efficacy and safety of tranexamic acid in reducing perioperative blood loss in patients with multilevel thoracic spinal stenosis: a retrospective observational study. Medicine (Baltimore) 2018;97:e13643.

24. Shakeri M, Salehpour F, Shokouhi G, et al. Minimal dose of tranexamic acid is effective in reducing blood loss in complex spine surgeries: a randomized double-blind placebo controlled study. Asian Spine J 2018;12:484-9.

25. Yoo JS, Ahn J, Karmarkar SS, Lamoutte EH, Singh $\mathrm{K}$. The use of tranexamic acid in spine surgery. Ann Transl Med 2019;7(Suppl 5):S172.

26. Winter SF, Santaguida C, Wong J, Fehlings MG. Systemic and topical use of tranexamic acid in spinal surgery: a systematic review. Global Spine J 2016;6:284-95.

27. Saberi H, Miri SM, Namdar MP. The effects of topically applied tranexamic acid on reduction of postlaminectomy hemorrhage. Tehran Univ Med J 2010;68:527-33.

28. Krohn CD, Sorensen R, Lange JE, Riise R, Bjornsen S, Brosstad F. Tranexamic acid given into the wound reduces postoperative blood loss by half in major orthopaedic surgery. Eur J Surg Suppl 2003;(588):5761.

29. Shen J, Yang Z, Fu M, Hao J, Jiang W. The influence of topical use of tranexamic acid in reducing blood loss on early operation for thoracolumbar burst fracture: a randomized double-blinded controlled study. Eur Spine J 2021;30:3074-80.

30. Wang X, Yang R, Sun H, Zhang Y. Different effects of intravenous, topical, and combined application of tranexamic acid on patients with thoracolumbar fracture. World Neurosurg 2019;127:e1185-9. 